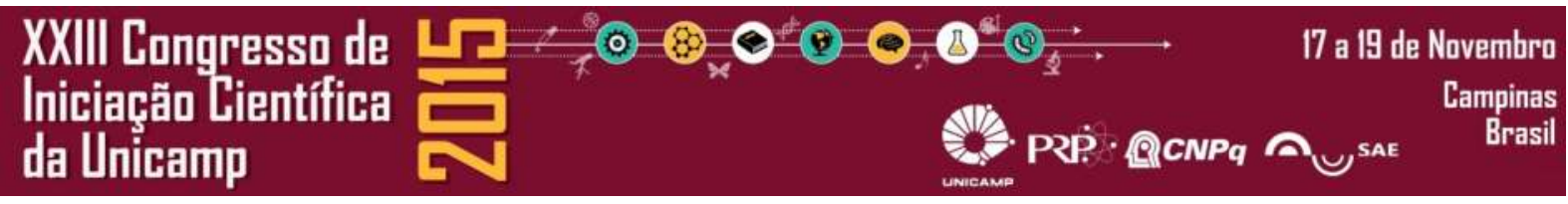

\title{
Computational study of the enantiomeric control of acetylation reactions by interaction with oligopeptides
}

\author{
lago T. de S. e Silva (IC), Leandro Martínez (PQ)
}

\begin{abstract}
Two systems containing a tetrapeptide in chloroform, one with and the other without a bisphenolic substrate were subjected to molecular dynamics simulations, and the number of hydrogen bonds formed between phenolic groups in the substrate and the catalyst was computed; it was found that the observed enantioselectivity contradicts the one found experimentally.
\end{abstract}

Key words: molecular dynamics; asymmetric catalysis; acetylation.

\section{Introduction}

Small polypeptides, being themselves asymmetric, may promote the emergence of asymmetries in non-chiral substrates, behaving effectively as small-scale enzymes (catalysts).

In this work, we made a computational study of the asymmetric acetylation of a bisphenolic compound (BIPH) in chloroform, which has been experimentally studied [1], assisted by a tetrapeptide (PAAS) as a catalyst in the reaction. Based on nuclear magnetic resonance (NMR) results concerning the transition state for the reaction, two mechanisms have been proposed: nucleophilic and base-assisted catalysis.

\section{Results and Discussion}

Two systems containing tetrapeptide PAAS were prepared, one with and one without The bisphenolic substrate (BIPH), both in chloroform. The systems were subjected to molecular dynamics simulations [2] using OPLS-AA [3] as the force field; the number of hydrogen bonds formed between the phenolic BIPH oxygens and PAAS was computed.

Image 1. PAAS-BIPH complex. One can clearly see the hydrogen bond between bisphenol and a sulphone in the catalyst, as well as the reactive nitrogen site in PAAS.

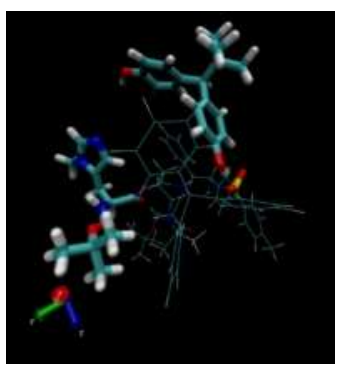

Table 1. Relative proportions (in \%) of hydrogen bonds made between the substrate's oxygens $(\mathrm{OA}$ and $\mathrm{OB})$ and some atom in PAAS $(\mathrm{x})$, and between the substrate and a nitrogen $(N)$ in the peptide associated with the nucleophilic attack in the proposed mechanisms [1].

\begin{tabular}{|l|l|l|l|l|}
\hline Sim. & X---OA & X---OB & N---OA & N---OB \\
\hline 1 & 42.13 & 57.87 & 100.00 & 0.00 \\
\hline 2 & 18.09 & 81.91 & 0.00 & 0.00 \\
\hline 3 & 19.41 & 80.59 & 100.00 & 0.00 \\
\hline 4 & 5.14 & 94.86 & 100.00 & 0.00 \\
\hline 5 & 24.11 & 75.89 & 37.29 & 62.71 \\
\hline Mean & 21.77 & 78.23 & 84.32 & 15.68 \\
\hline
\end{tabular}

\section{Conclusions}

Analysis of the simulation results showed that there is a systematic tendency of the peptide to complex with the substrate in a way that directly contradicts the experimental results, in the context of the bifunctional hypothesis [1]; the simulations suggest that the reaction would wield mainly the opposite enantiomer than the one observed experimentally.

\section{Acknowledgement}

The author acknowledges support from Conselho Nacional de Desenvolvimento Científico e Tecnológico (CNPq).

${ }^{1}$ Lewis, C. A. et alia. J. Am. Chem. Soc. 2008, 130, 16358.

${ }^{2}$ Frenkel, D.; Smit, B. Understanding Molecular Simulation, $2^{\text {nd }}$ ed.; Academic Press: San Diego, 2001; pp 63-71.

${ }^{3}$ Jorgesen, W. L.; Mazwell, D. S.; Tirado-River, J. J. Am.

Chem. Soc. 1996, 118, 11225. 